\title{
Experimental Verification of a Completely Soft Gripper for Grasping and Classifying Beam Members in Truss Structures
}

\author{
Lili Bykerk and Dikai Liu ${ }^{1}$
}

\begin{abstract}
Robotic object exploration and identification methods to date have attempted to mimic human Exploratory Procedures (EPs) using complex, rigid robotic hands with multifaceted sensory suites. For applications where the target objects may have different or unknown cross-sectional shapes and sizes (e.g. beam members in truss structures), rigid grippers are not a good option as they are unable to adapt to the target objects. This may make it very difficult to recognise the shape and size of a beam member and the approaching angles which would result in a secure grasp. To best meet the requirements of adaptability and compliancy, a soft robotic gripper with simple exteroceptive force sensors has been designed. This paper experimentally verifies the gripper design by assessing its performance in grasping and adapting to a variety of target beam members in a truss structure. The sensor arrangement is also assessed by verifying that sufficient data is extracted during a grasp to recognise the approaching angle of the gripper. Firstly, the gripper is used to grasp each beam member from various angles of approach and readings from the force sensors are collected. Secondly, the collected sensor data is used to train and then test a range of commonly used classifiers for classification of the angle of approach. Thirdly, the classification results are analysed. Through this process, it is found that the gripper is proficient in grasping the variety of target beam members. Despite the uncertainty in the gripper pose, the sensor data collected from the soft gripper during a grasp is sufficient for classification of the angles of approach.
\end{abstract}

\section{INTRODUCTION}

There are many complex elements of truss structures (such as power transmission towers) which make climbing the structures difficult. Variations in the cross-sectional shape, size and orientation of beam members and the presence of structural fixtures form a difficult environment for a robot to climb. For safe climbing, robot grippers should be able to:

- reliably and securely grasp the variety of cross-sectional shapes and sizes of beam members (known shapes are "L", "O" and possibly many other shapes).

- adapt to uneven surfaces where mating plates, bolts and other structural fixtures are present.

- incorporate sensing technology to extract features (such as the cross-sectional shape, size and orientation) of target beam members during a grasp.

Developing a robotic gripper for the application of climbing a truss structure is challenging, since there are desired design features which contradict each other; it is difficult to design a compliant, adaptive gripper which is also capable

\footnotetext{
${ }^{1}$ Lili Bykerk and Dikai Liu are with Centre for Autonomous Systems, School of Elec, Mech and Mechatronic Systems, University of Technology Sydney, 81 Broadway, Sydney, Australia. Email: lili.bykerkestudent.uts.edu.au, dikai.liu@uts.edu.au
}

of high grasping forces. Rigid grippers provide the greatest strength in grasping, with little adaptability to variations in object shape. These grippers commonly take the form of parallel mechanisms and have been used in many climbing robots [1]-[3] due to their strength and reliability of grasping in known environments. Underactuated and adaptive grippers are more proficient in adapting to varying shapes and sizes of objects, but typically have lower grasping strength than completely rigid grippers. They are also limited in their adaptive capabilities due to the rigid links comprising the gripper's fingers. Examples in recent literature [4], [5] are able to reliably grasp and adapt to a range of lightweight household objects. Soft robotic grippers have the benefits of inherent compliancy and adaptability, however, also lack grasping strength. These grippers have also generally been designed with the target application of grasping small and lightweight (typically household) objects [6]-[8]. To the best of our knowledge, no soft gripper has been designed for the intended application of a robot to climb truss structures.

Roboticists have naturally looked to the human hand for inspiration when developing grippers with sensory suites capable of performing complex exploratory motions. Research into human haptic perception ("the process of perceiving the characteristics of objects through touch" [9]) has proved that humans use certain "Exploratory Procedures" (EPs) to extract desired features and information about target objects [10], [11]. By using a relatively fast EP such as enclosure (grasping), information about the target object can be extracted in a short time period ("haptic glance" [12]).

Robotic haptic perception is a challenging concept. Implementation issues such as installing tactile arrays in grippers, errors in actuation and sensing and motion restrictions due to hand and arm dexterity complicate the process. To execute exploratory motions, complex control systems are also typically required. Recent research in robotic haptic perception has focused on identifying household objects by implementing EPs [13]-[18]. Current state of the art robots are incapable of identifying objects and their properties with human accuracy.

Due to the simplicity of the concept, the haptic glance [12] is much more easily appropriated to the robotic sense. By using a haptic glance as a method of exploration, the hardware set-up, including the actuation method can be simple and sensors can be sparse. Inspired by the concept of the haptic glance, a two fingered adaptive, underactuated hand with an array of 8 simple barometric pressure sensors per finger was designed for object identification [19]. The hand was used to grasp 11 different household items 20 times 
in both constrained and unconstrained orientations. 100\% classification accuracy of the object type was reported for the constrained orientations and $94.32 \%$ accuracy for the unconstrained orientations using a Random Forest (RF) classifier. Using a parametric method with the RF classifier for the unconstrained orientations achieved $100 \%$ classification.

The concept of haptic perception in soft robotics is in its early stages of development. One major challenge is the lack of knowledge of a completely soft gripper's configuration during a grasp. In an attempt to address the forward kinematics problem, a soft robotic gripper with proprioception was designed [20]. The gripper, comprised of three modified pneumatic-network fingers fitted with resistive bend sensors was used to perform haptic identification of objects using only one data point from each of the three fingers during a haptic glance. Using a k-nearest neighbours (k-NN) classifier, the classification results showed that objects of a certain test set could be classified with $98 \%$ accuracy using enveloping grasps and $68 \%$ with pinch grasps. In an alternative approach, a modular, reusable sensor skin customisable and scalable to arbitrary surfaces was developed [21]. The sensor skin, comprised of one bend sensor and one strain sensor was attached to a soft pneumatic actuator gripper with three individually controlled fingers. The soft gripper was capable of visualising an object's shape by obtaining an outline through knowledge of the pose of the actuator and the fingertip contact.

For a robot to climb a truss structure such as a power transmission tower, a soft gripper would provide the required compliancy and adaptability for making adequate contact with various target members for reliable grasping. Simple force sensors retrofitted to the gripper's finger surfaces can provide data for feature extraction during grasping. Ideally, the desired features would be extracted by using the most efficient method of haptic exploration (a haptic glance). It is understood that a completely soft gripper lacks the required grasping strength for a robot to climb a truss structure. For this reason, it is envisaged that the soft gripper described in this paper can be placed inside a mechanical frame which provides the grasping strength. The design of the mechanical frame is out of the scope of this paper.

In this paper, the design of a soft gripper is verified by assessing its performance in grasping a variety of target members and collecting sufficient sensor data for classifying the gripper's angle of approach. The design is evaluated by performing haptic glances to collect data at many angles of approach to various target members. The data is then used to train a range of commonly used classifiers, with the goal of classifying the angle of approach of the gripper against the various target members. Finally, the classification results are used to assess the design of the gripper.

\section{GRIPPER DESIGN}

\section{A. Soft Gripper}

To meet the functional requirements for the application environment, the soft gripper design is similar to the topology optimised material distribution of our previous design [22].

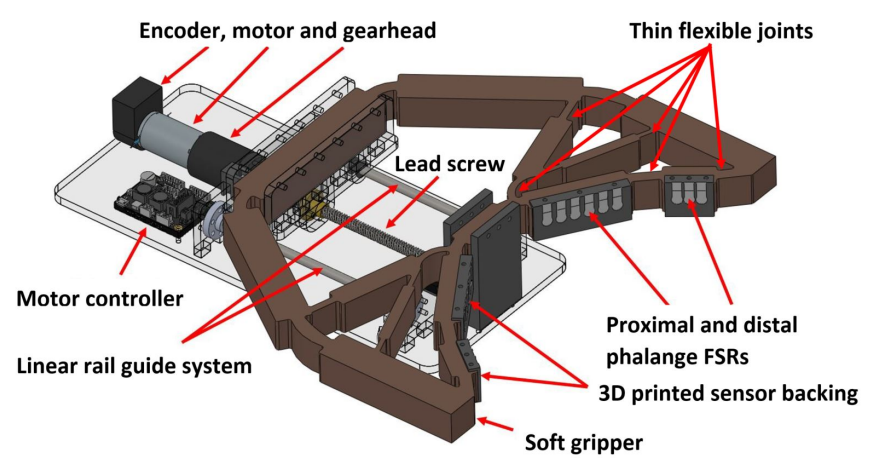

(a)

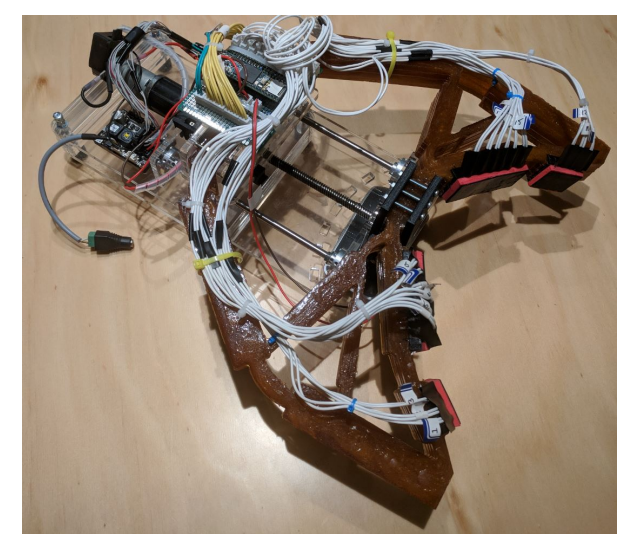

(b)

Fig. 1. Gripper design a) CAD design of gripper with actuator and sensors b) Physical gripper with controller, actuator and sensors wired.

However, some design modifications (see Fig. 1) have been made to 1) create more defined proximal and distal phalanges, 2) add thin flexible joints to increase the compliancy of the structure and reduce the required actuation force and 3) scale up the design to enable grasping of a larger range of sizes of beam members.

By using flexible and elastomeric materials, soft robotic grippers have the benefits of inherent compliancy and adaptability; making the gripper ideal for grasping a variety of cross-sectional shapes and sizes of beam members. These properties also make it very difficult to measure joint angles, calculate finger positions and achieve strength in grasping. The gripper is prone to warping and bending due to factors such as gravity, soft material rigidity and the points of contact during a grasp. The gripper in this paper does not incorporate any joint sensing, only exteroceptive force sensing (using Force Sensitive Resistors (FSRs)) and linear distance sensing (using a motor encoder). For the practical application of a robot climbing a truss structure, the grippers will require an external rigid mechanical mechanism to provide strength in grasping, whilst still permitting the soft inner sections to deform and adapt to the target beam members.

\section{B. Actuation}

An actuator is required to actuate the gripper for grasping a target beam member. In this design, a linear actuator 
consisting of a maxon A-max DC motor with planetary gearhead and encoder, coupled to a lead screw as shown in Fig. 1 is used. The motor drove the system back and forth along the linear rail to open and close the gripper's fingers. At the extremities of the linear actuator stroke were two limit switches. The data (18 FSR analog values, 2 limit switch states, motor current, encoder reading) was streamed over serial for recording. The motor current and limit switch states were monitored by the control system for driving the gripper open, closed, or to a stop.

\section{Force Sensors}

The exteroceptive sensing consisted of an array of 18 small, off the shelf Interlink FSRs (FSR 400 Short). Six sensors were placed on each of the proximal phalanges and three on each of the distal phalanges of the two fingers as shown in Fig. 1a. These FSRs were selected as they could be easily retrofitted to the soft gripper in an arrangement that was anticipated would provide sufficient grasp data. Typically, FSRs do not function well without a rigid backing surface. 3D printed sections were used to both secure the sensors to the inner finger surfaces and to ensure that the sensors were evenly spaced along the surface of the fingers. The 3D printed sensor backing also allowed for reliable and repeatable readings due to its rigidity. Because of the sparseness of the sensor distribution, in certain scenarios during grasping it was possible for a contact to occur directly between two sensors. In this scenario, the sensors on either side of the contact would not register a force. To aid in force distribution to overcome this issue, the FSRs were covered with a $3 \mathrm{~mm}$ layer of PE-180 foam and topped with a 1 $\mathrm{mm}$ thick section of rubber sheet. All layers were adhered together using $3 \mathrm{M}$ clear double sided tape. A cross-section of the final assembly is shown in Fig. 2.

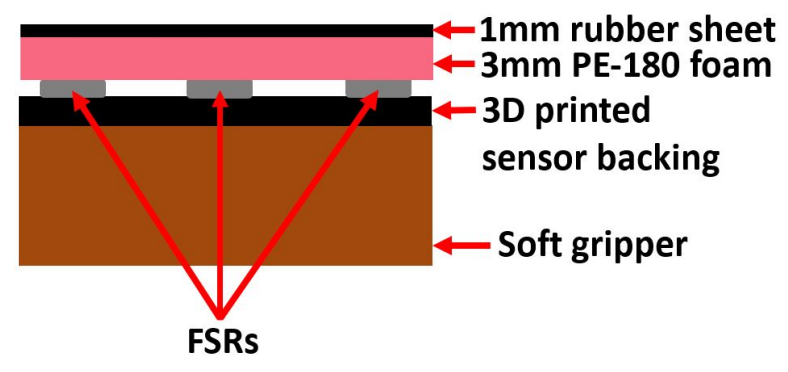

Fig. 2. Cross-section of the sensor assembly.

\section{DESIGN OF EXPERIMENT}

This section provides the description of the experiments, including the target object set used and the data extraction methods.

\section{A. Target Objects}

The set of target objects used in the experiments consisted of three sizes of "L" shaped beam members and three other shapes of beam members of varying sizes as shown in Fig. 3 and listed in Table I. All of the "L" shaped beam members

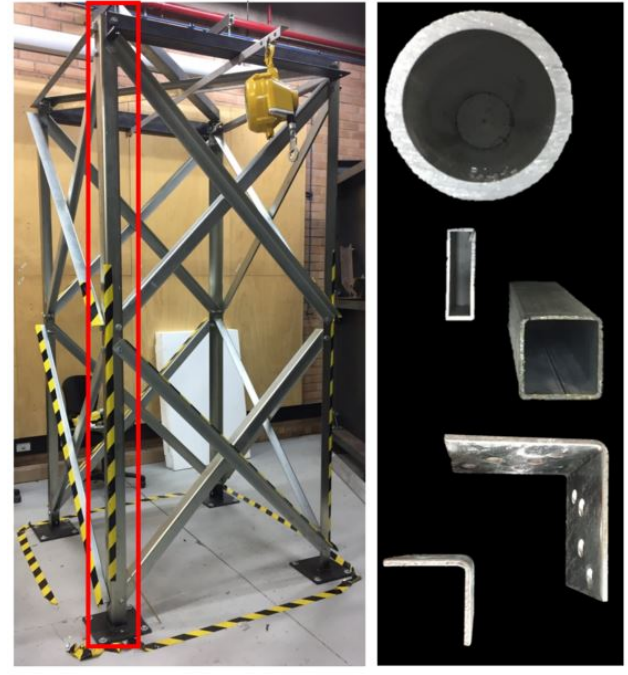

Fig. 3. Beam members in the target object set. Lab test rig - cage section with $75 \times 75 \times 6$ " $L$ " shaped target beam member highlighted (left), other target beam members (right).

are of dimensions typically found in some $66 \mathrm{kV}$ transmission towers. The angles of approach (grasping angles) for the "L" shaped beam members are defined in Fig. 4.

TABLE I

Dimensions of Beam Members in the Target Object Set.

\begin{tabular}{ccc}
\hline $\begin{array}{c}\text { Beam } \\
\text { shape }\end{array}$ & $\begin{array}{c}\text { Cross-sectional } \\
\text { dimensions (mm) }\end{array}$ & $\begin{array}{c}\text { Wall thickness } \\
(\mathbf{m m})\end{array}$ \\
\hline "L" & $50 \times 50$ & 4 \\
"L" & $75 \times 75$ & 6 \\
"L" & $100 \times 100$ & 4 \\
"O" & $\varnothing 63$ & 6 \\
Square & $51 \times 51$ & 2.5 \\
Rectangular & $81 \times 25$ & 3 \\
\hline
\end{tabular}

\section{B. Experimental Rig}

The experimental rig consisted of a beam member from the target object set, gripper with linear actuator and angle measurement tools. The angle measurement tool was fixed to the target beam member and was used to perform repeatable grasps at a given angle and distance from the surface of the beam members. These tools consisted of a circular structure with notches at $10^{\circ}$ increments, located at varying radii from the centre of the target beam members. These notches were used to lock the linear actuator to the angle measurement tool at the desired angle of approach for data collection.

\section{Experiment Procedure}

Depending on the cross-sectional shape and symmetry of the target beam members, different angles of approach were used for data collection in $10^{\circ}$ increments. For the "L" shaped members, data was collected over $360^{\circ}$ (see Fig. 4), for the square shaped member, $90^{\circ}$, for the rectangular shaped member, $180^{\circ}$ and for the "O" shaped member a single angle of approach. 


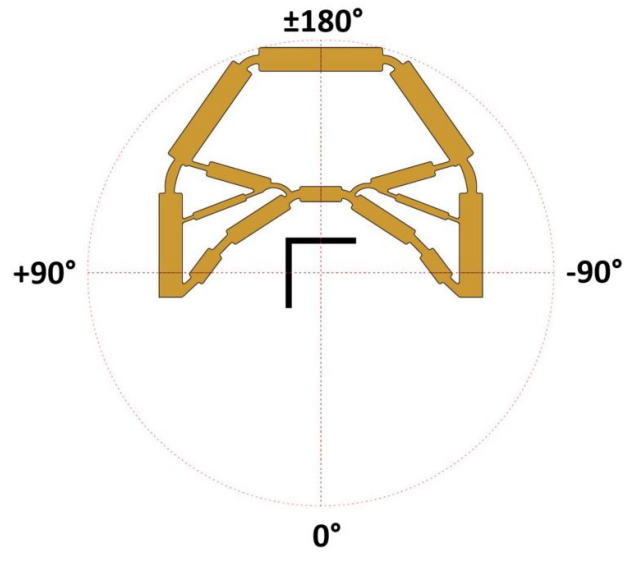

Fig. 4. Angles of approach for the "L" shaped target beam members.

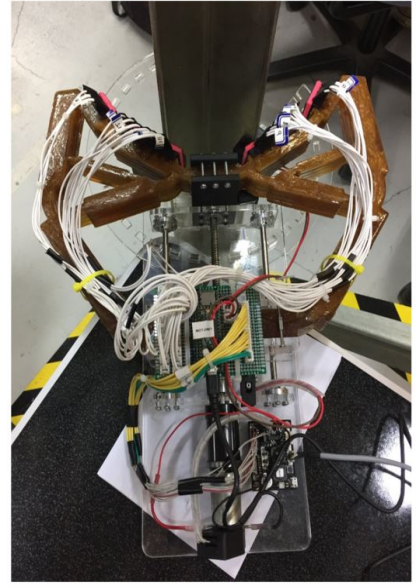

(a)

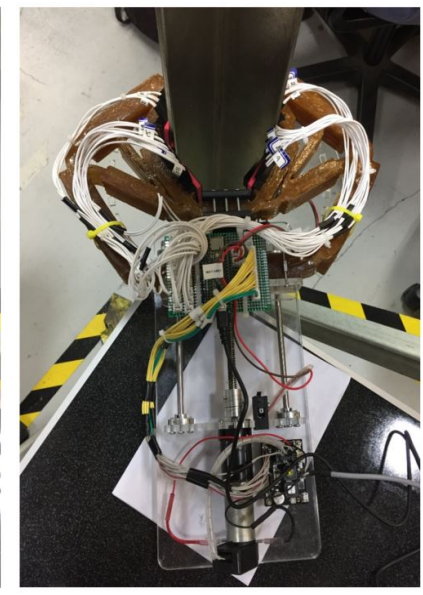

(b)
Fig. 5. Gripper pose during data collection, positioned at $-40^{\circ}$ on a $75 \times 75 \times 6$ "L" shaped beam member (a) fully opened, (b) fully closed.

In the experiments, the soft gripper approaches a beam face perpendicularly, with gripper sag due to gravity being observed. The angle measurement tool was attached to a target beam member and the gripper was manually positioned to the desired angle by locking the linear actuator base into the angle measurement tool. When the data collection procedure began, the motor would drive the gripper completely open (until the low limit switch of the linear actuator was triggered). At this point the encoder would zero and the gripper would then close and open ten times. The FSR sensor data was recorded at the end of each grasp; defined by one of two conditions - either the upper limit switch had been triggered (which defined the limit of the linear actuator stroke length) or a user defined motor current limit $(325 \mathrm{~mA})$ had been reached. This process was repeated by first incrementing the angle of approach by $10^{\circ}$ and then performing the data collection. Fig. 5 shows the gripper at two time instances (fully open and fully closed states) during data collection at $-40^{\circ}$ for a "L" shaped beam member.

\section{DATA Collected}

\section{A. Raw Data}

Fig. 6 shows averaged raw data from the 10 repeated grasps performed at angles of approach from $-10^{\circ}$ to $-90^{\circ}$ in $10^{\circ}$ increments for two of the "L" shaped beam members from the target object set $(75 \times 75 \times 6$ and $100 \times 100 \times 4)$. For each of the angles of approach shown, unique FSR distribution patterns can be observed. This observation generally extends to the remaining angles of approach to beam members from the target object set, with the exception of the angles of approach discussed in Sec. V-B.

For all of the beam members in the target object set, the averaged raw data across all of the angles of approach showed that sensors 10 and 12 were almost completely redundant. However, the remainder of the sensors were typically making contact with the target beam members across all angles of approach.

\section{B. Classification Algorithms}

To evaluate the gripper design, the collected FSR sensor data was analysed to verify the gripper's adaptability to various target beam members. The sensor arrangement was also assessed by verifying that the sensor data was sufficient for recognising the angle of approach during a grasp. In the verification, the sensor data was used to train and evaluate a variety of commonly used classifiers, including k-nearest neighbours (k-NN), Linear Discriminant Analysis (LDA), Multiclass Support Vector Machine (SVM), Naïve Bayes, Bagged Trees Ensemble and Random Forests (RF).

The k-NN was trained with 2 neighbours, an Euclidean distance metric and an inverse distance weight. The Multiclass SVM was trained using several different Kernel types, with Gaussian giving the best results. Random Forests were trained with 10,100 and 1000 trees to determine the forest size which would provide a suitable trade-off between accuracy and computational power. For each of the different classifiers, the results were averaged over 1000 rounds.

\section{RESUlTS AND DisCUSSION}

\section{A. Factors Affecting Data Precision}

Despite efforts to ensure that the gripper's angle of approach was consistent for all repeated grasps, slight misalignments in the rig components could have introduced variance in FSR readings and hence FSR distribution patterns.

Natural wear on the linear actuator components occurred over time, with the lead screw and nut of the linear actuator rig becoming visibly worn down. The main cause of this wear was the elastic restoring force from the soft gripper as it was actuated into the closed position; causing misalignment and grinding. These components were not replaced during the data collection and could have resulted in slightly different distance measurements being recorded by the motor encoder as the component wear worsened.

Sensor drift over time can affect the reliability of the sensor readings, however due to the short time period in which the data collection took place, this drift offset was not 


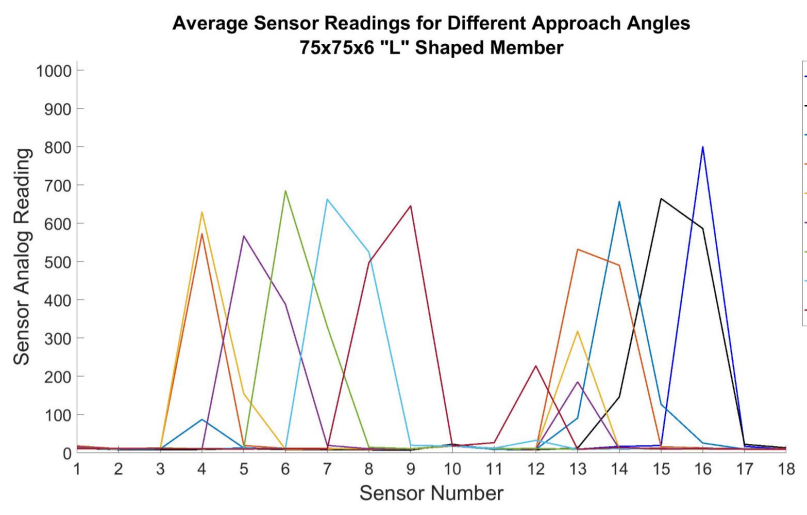

(a)

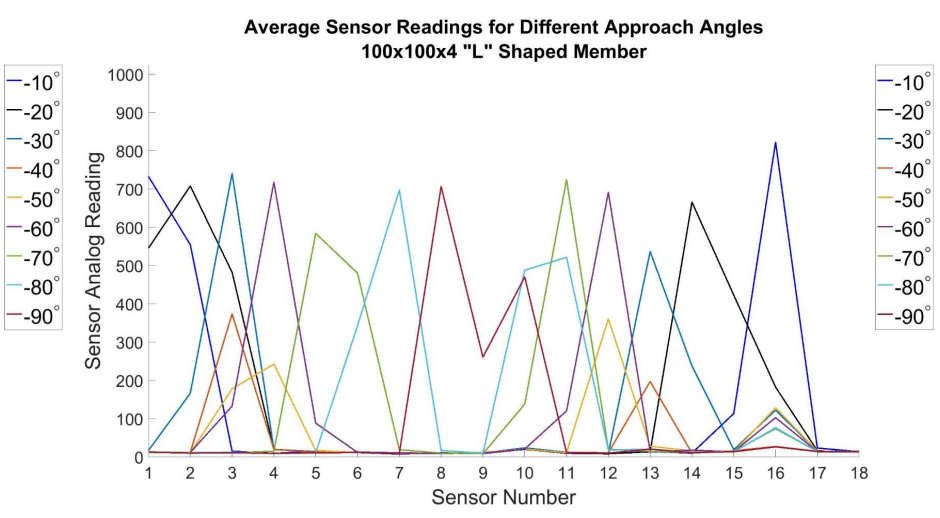

(b)

Fig. 6. Averaged raw FSR readings (from 10 repeated grasps) across 9 angles of approach $\left(-10^{\circ}\right.$ to $-90^{\circ}$ in $10^{\circ}$ increments) for two beam members from the target object set. (a) 75x75x6 "L" shaped beam member, (b) 100x100x4 "L" shaped beam member.

considered. For more precise results over longer periods of time, sensor drift should be considered.

A selection of sensors were subject to repetitive forces from edges of the beam members during data collection. Some of these sensors degraded significantly in their sensitivity and needed replacement. This is a potential factor that has reduced the precision of the overall data collected. Calibration was not performed to take this into consideration, since it was expected to have some variance and fluctuation in the sensor readings regardless.

\section{B. Raw Data Observations}

Throughout the data collection process, it was immediately clear that there would be certain angles of approach to various target beam members which would result in the collection of insufficient data for unambiguous classification of the angle of approach. When grasping at a series of angles of approach $\left(-100^{\circ}\right.$ to $\left.-140^{\circ}\right)$ for the $50 \times 50 \times 4$ "L" shaped beam member and angles of approach $\left(-10^{\circ},-170^{\circ}, \pm 0^{\circ}\right)$ for the $81 \times 25 \times 3$ rectangular shaped beam member, the linear actuator reached the extremity of its stroke length and the gripper's fingers were not contacting any surfaces of the target beam members. Another scenario with limited data was observed when a major edge of a target beam member fell between the proximal and distal finger sections (where no sensors were located) during a grasp.

Certain angles of approach for different target beam members were also observed to have yielded very similar FSR and motor encoder distance readings. This was expected to occur when grasping " $\mathrm{L}$ " shaped beam members at the orientations of $+90^{\circ}$ and $\pm 180^{\circ}$, since at these angles of approach, the gripper was grasping at two edges, equidistant from the axis of symmetry of the gripper. However, due to one edge having a greater surface area than the other, unique FSR distribution patterns were generally recorded, and the FSR distribution patterns of $+90^{\circ}$ and $\pm 180^{\circ}$ were simply mirror images of each other. In some cases, due to slight misalignment in the angle of approach, the FSR distribution patterns on both fingers appeared identical.
For these scenarios, it was anticipated that these angles of approach would produce erroneous results when run through the trained classifiers, since the data could be easily confused by visual inspection alone.

\section{Results of Classification}

The raw data obtained in the data collection phase (18 FSR analog and motor encoder distance readings) was used to train and evaluate the performance of the various classifiers, without any data pre-processing. The classifiers were trained with data from 6 of the 10 grasps at each angle of approach, with the remaining 4 grasps used for test validation. The classifiers listed in Table II were also evaluated using 10fold cross-validation to reduce the effect of a specific test dataset on the classifier performance. The RF classifier results listed in Table III were similarly evaluated without bias by calculating the out-of-bag (OOB) error [23].

TABLE II

ClASSIFIER RESUlTS - GRIPPER ANGLE OF APPROACH.

\begin{tabular}{ccc}
\hline Classifier & $\begin{array}{c}\text { Average 10-fold } \\
\text { Cross-validation } \\
\text { Accuracy (\%) }\end{array}$ & $\begin{array}{c}\text { Average Test } \\
\text { Data Accuracy (\%) }\end{array}$ \\
\hline k-NN & 96.58 & 96.22 \\
LDA & 95.16 & 95.22 \\
Multiclass SVM & 95.02 & 95.52 \\
Naïve Bayes & 93.50 & 93.66 \\
Bagged Trees Ensemble & 94.90 & 94.13 \\
\hline
\end{tabular}

TABLE III

RF RESULTS - GRIPPER ANGLE OF APPROACH.

\begin{tabular}{ccc}
\hline $\begin{array}{c}\text { Number of Trees } \\
\text { in Random Forest }\end{array}$ & $\begin{array}{c}\text { Average OOB } \\
\text { Error (\%) }\end{array}$ & $\begin{array}{c}\text { Average Test } \\
\text { Data Accuracy (\%) }\end{array}$ \\
\hline 10 & 20.54 & 94.43 \\
100 & 4.88 & 96.16 \\
1000 & 4.48 & 96.23 \\
\hline
\end{tabular}

The results in Table II and Table III show that high classification accuracies were achieved by all of the classifiers, 
even with the factors and scenarios (stated in Sec. V-A and Sec. V-B) that affect the data precision. For the RF classifier, growing at least 100 trees in the forest provided an acceptable trade-off between classification accuracy and computational effort. Whilst the classification results are promising overall, there are some limitations to the overall classification accuracy, as seen in Fig. 7 with the misclassification of the ambiguous angles of approach.

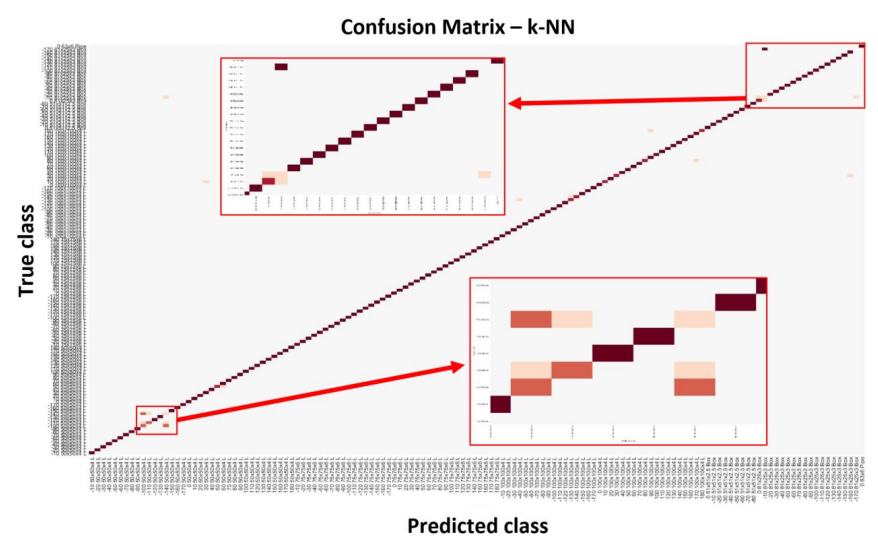

Fig. 7. Example confusion matrix for one trained k-NN classifier. Lower zoomed box shows the confusion at angles of approach of $-100^{\circ}$ to $-140^{\circ}$ for the 50x50x4 "L" shaped beam member. Upper zoomed box shows the confusion at angles of approach of $-170^{\circ},-10^{\circ}$ and $0^{\circ}$ for the $81 \times 25 \times 3$ rectangular beam member.

\section{CONClusions And Future Work}

This research has verified that the soft gripper designed for a truss structure climbing robot was able to reliably grasp different cross-sectional shapes and sizes of beam members from the target object set. The gripper generally achieved good surface contact for sufficient data collection during a grasp at varying approach angles. The collected data was used to train classifiers to determine the angle of approach of the gripper to a target beam member. All classifiers achieved good classification results, with marginal variance in accuracy across the different classifiers.

Future work includes increasing the classification accuracy, identifying the geometric shape of a beam member by minimising the number of required touches and finding the ideal angle of approach for a grasp on a structural beam member.

\section{ACKNOWLEDGEMENT}

This research is supported in part by the Centre for Autonomous Systems at the University of Technology Sydney, Australia and the Tokyo Electric Power Company, Japan.

\section{REFERENCES}

[1] C. Balaguer, A. Gimenez and C.M. Abderrahim, "ROMA Robots for Inspection of Steel Based Infrastructures," Industrial Robot: An International Journal, vol. 29, no. 3, pp. 246-251, 2002.

[2] M. Tavakoli, A. Marjovi, L. Marques, A. T. de Almeida, "3DCLIMBER: A Climbing Robot for Inspection of 3D Human Made Structures," IEEE/RSJ International Conference on Intelligent Robots and Systems (IROS), pp. 4130-4135, IEEE, 2008.
[3] Y. Guan, L. Jiang, H. Zhu, X. Zhou, C. Chuanwu, W. Wu, Z. Li, H. Zhang and X. Zhang, "Climbot: A Modular Bio-inspired Biped Climbing Robot," IEEE/RSJ International Conference on Intelligent Robots and Systems, pp. 1473-1478, IEEE, 2011.

[4] A. M. Dollar and R. D. Howe, "The Highly Adaptive SDM Hand: Design and Performance Evaluation," The International Journal of Robotics Research, vol. 29, no. 5, pp. 585-597, 2010.

[5] L. U. Odhner, L. P. Jentoft, M. R. Claffee, N. Corson, Y. Tenzer, R. R. Ma, M. Buehler, R. Kohout, R. D. Howe and A. M. Dollar, "A Compliant, Underactuated Hand for Robust Manipulation," The International Journal of Robotics Research, vol. 33, no. 5, pp. 736$752,2014$.

[6] R. Deimel and O. Brock, "A Compliant Hand Based on a Novel Pneumatic Actuator," IEEE International Conference on Robotics and Automation (ICRA), pp. 2047 - 2053, IEEE, 2013.

[7] M. Manti, T. Hassan, G. Passetti, N. d'Elia, C. Laschi and M. Cianchetti, "A Bioinspired Soft Robotic Gripper for Adaptable an Effective Grasping," Soft Robotics, vol. 2, no. 3, pp. 107-116, 2015.

[8] M.E. Giannaccini, I. Georgilas, I. Horsfield, B. H. P. M. Peiris, A. Lenz, A. G. Pipe and S. Dogramadzi, "A Variable Compliance, Soft Gripper," Autnonomous Robots, vol. 36, no. 1-2, pp. 93-107, 2014.

[9] A. El Saddik, M. Orozco, M. Eid and Jongeun Cha, "Haptics: General Principles," Springer Series on Touch and Haptic Systems, Springer Berlin Heidelberg, pp. 1-20, 2011.

[10] S.J. Lederman and R.L. Klatzky, "Hand movements: A window into haptic object recognition," Cognitive Psychology, vol. 19, no. 3, pp.342-368, 1987.

[11] S.J. Lederman and R.L. Klatzky, "Extracting Object Properties Through Haptic Exploration," Acta Psychologica, vol. 84, no. 1, pp. 29-40, 1993.

[12] R.L. Klatzky and S.J. Lederman, "Identifying Objects from a Haptic Glance," Perception \& Psychophysics, vol. 57, no. 8, pp. 1111-1123, 1995.

[13] V. Chu, I. McMahon, L. Riano, C.G. McDonald, Q. He, J.M. Perez-Tejada, M. Arrigo, N. Fitter, J.C. Nappo, T. Darrell and K.J. Kuchenbecker, "Using Robotic Exploratory Procedures to Learn the Meaning of Haptic Adjectives," 2013 IEEE International Conference on Robotics and Automation (ICRA), pp. 3048-3055, 2013.

[14] B. Higy, C. Ciliberto, L. Rosasco and L. Natale, "Combining sensory modalities and exploratory procedures to improve haptic object recognition in robotics," in Proceedings 2016 IEEE-RAS 16th International Conference on Humanoid Robots (Humanoids), pp. 117-124, 2016.

[15] N.F. Lepora, K. Aquilina and L. Cramphorn, "Exploratory Tactile Servoing With Active Touch," IEEE Robotics and Automation Letters, vol. 2, no. 2, pp. 1156-1163, 2017.

[16] U. Martinez-Hernandez, T.J. Dodd, L. Natale, G. Metta, T.J. Prescott and N.F. Lepora, "Active contour following to explore object shape with robot touch," 2013 World Haptics Conference (WHC), pp. 341346, 2013.

[17] U. Martinez-Hernandez, T.J. Dodd and T.J. Prescott, "Feeling the Shape: Active Exploration Behaviors for Object Recognition With a Robotic Hand," IEEE Transactions on Systems, Man, and Cybernetics: Systems, vol PP, no. 99, pp. 1-10, 2017.

[18] N. Sommer and A. Billard, "Multi-contact haptic exploration and grasping with tactile sensors," Robotics and Autonomous Systems, vol. 85, pp. 48-61, 2016.

[19] A.J. Spiers, M.V. Liarokapis, B. Calli and A.M. Dollar, "Single-Grasp Object Classification and Feature Extraction with Simple Robot Hands and Tactile Sensors," IEEE Transactions on Haptics, vol. 9, no. 2, pp. 207-220, 2016

[20] B.S. Homberg, R.K. Katzschmann, M.R. Dogar and D. Rus, "Haptic identification of objects using a modular soft robotic gripper," 2015 IEEE/RSJ International Conference on Intelligent Robots and Systems (IROS), pp. 1698-1705, 2015.

[21] B. Shih, D. Drotman, C. Christianson, Z. Huo, R. White, H.I. Christensen and M.T. Tolley, "Custom soft robotic gripper sensor skins for haptic object visualization," 2017 IEEE/RSJ International Conference on Intelligent Robots and Systems (IROS), pp. 494-501, 2017.

[22] L. Bykerk, D. Liu and K. Waldron, "A Topology Optimisation Based Design of a Compliant Gripper for Grasping Objects with Irregular Shapes," 2016 IEEE International Conference on Advanced Intelligent Mechatronics (AIM), pp. 383-388, 2016.

[23] L. Breiman, "Random Forests: Leo Breiman and Adele Cutler", stat.berkeley.edu, [Online]. Available: www.stat.berkeley.edu/ breiman/RandomForests/cc_home.htm [Accessed Feb. 27, 2018]. 